\title{
Research on Three Typical Methods of Infrared Weak Small Targets Background Suppression under Cloud Background
}

\author{
Zhongmin $\mathrm{LI}^{1, a}$, Guowei ZOU ${ }^{2, b}$, Lifei MEl ${ }^{3, c}$ \\ ${ }^{1}$ School of Information Engineering, Nanchang Hangkong University, Nanchang, 330063, China \\ ${ }^{2}$ School of Information Engineering, Nanchang Hangkong University, Nanchang, 330063, China \\ ${ }^{3}$ School of Information Engineering, Nanchang Hangkong University, Nanchang, 330063, China \\ aemail: zhongmli@163.com, bemail: 824206587@qq.com, cemail: 851140348@qq.com
}

Keywords: Background Suppression; Infrared Image; Cloud Background

\begin{abstract}
Background suppression of weak small targets in infrared image is the key of image tracking and monitoring, especially under the cloud background. In the area of image signal processing, there are a lot of background suppression methods can be used to image filter by combining the characteristics of infrared image under the cloud background. In this paper, we introduce three typical background filter methods such as pulse median filter, multi-structural morphological filter and wavelet threshold method, realize them using MATLAB, and analyze their performances of background suppression of weak small targets in infrared image under the cloud background.
\end{abstract}

\section{Introduction}

Background suppression of weak small targets in infrared image is the key of image tracking and monitoring, especially under the cloud background. Various image preprocessing algorithms are used for the target enhancement of infrared image and background suppression, in order to improve the SNR of image. Infrared image background generally has relevance in the low-frequency domain of the image, and the target in the background is generally located in the high-frequency portion of the image in the frequency domain. Therefore, the image background can be suppressed directly, and it can also be predicted to realize the background suppression by calculating the residual image.

Median filter is an image signal filter technology in the sort of statistics presented on the basis of theoretical principles. Suppressing noise (especially impulse noise) can save target signal edge, and acute angle details. Top-hat transform algorithm (Top-hat) [1] is attributed to a non-linear filter, which effect is better than the median filter during the image background suppression, but we need the priori knowledge of image before making the top-hat transform for it. In the image signal processing point of view, its adaptability is weak. Mohiy found the two-dimensional least mean square filter (TDLMS) [2]. Its main application is two-dimensional linear prediction in the background of the image, and after subtracting the estimated background image with the original image, in order to enhance the signal to noise ratio. Lee et al [3] found a weighted low-pass filter method, which would not only make images smoother, but also save the details of the target image edge, but its applicability and versatility relatively are poor. Zeng [4] found a median filter based on bilateral, introduced in the background filter process template to enhance the image of inhibition effect, but these methods required the actual background to create a template.

Mathematical Morphology background suppression primarily using morphological structure elements are not the same as opening or closing operation to suppress the background of the image. It is firstly to define structural element (filter window) of the image signal to match, in order to better extract destination information, and then to suppress noise. Mathematical morphology filter [5] [6] is a relatively common for small target image background suppression methods. It firstly analyzes the relationship between the pixel and pixels in the image, and then to take a set of operators such as dilation, erosion, opening, and closing. Many domestic and foreign researchers apply Top-hat transform to the field of infrared image background suppression [7] [8]. 
Morphological reconstruction filter [9] belongs to the important morphological filter algorithm for a class while processing the binary. If you do not have a target in the presence of the marker images, do not restore the image, on the contrary, the image can be completely recovered. Morphological reconstruction filter is not sensitive to noise, while suppressing the background, does not produce a new signal noise [10], and does not change the position of the image [11]. Ref. [12] used morphological reconstruction filter to implement infrared image background suppression, which got better results than Top-Hat transformation. Ref. [13] proposed an infrared image based on morphology background suppression algorithm, which was realized in the FPGA platform.

Wavelet transform method for image background suppression belongs to image transform domain method. In 1986, Mallat [14] was the first one found the fast algorithm to signal decomposition and reconstruction of the wavelet transform algorithm based on the classical fourier transform. After that, Donoh and J.Sotue et al. found the soft threshold wavelet transform and the hard threshold wavelet transform [15] [16] image background suppression methods. They deduced formulas traditional wavelet threshold, and made it confirmed that its proposed threshold was the best one. This year, Donoh and Coifrnan studied a translation invariant wavelet background suppression algorithm [17]. According to this principle, Johnstone [18], who focused on adaptive thresholding wavelet transform with a translation invariant background fusion inhibition, discovered a face image spatial wavelet threshold background suppression method, which chose the threshold according to the statistical properties of the image shift. Zhao found a complex algorithm based on wavelet transform local domain. Luisier [19] found that a ladder operation without relying on wavelet transform background suppression method.

In this paper, we choose three typical background filter methods such as pulse median filter, multi-structural morphological filter and wavelet threshold method as examples to compare their performances of background suppression of weak small targets in infrared image under the cloud background.

\section{Pulse Median Filter}

There are mainly two steps in the pulse detection algorithm based on adaptive median filter on cloud background infrared image background suppression. The first step is to use the extreme value method for cloud background image processing by using the structure information between neighboring pixels, which set an appropriate threshold value to detect information signals or noises points; the second step is to use the noise point estimate of different regions in the image based on noise interference to adaptively adjust the window size, and then use the pulse median filter based on impulse noise to realize background suppression.

\section{Multi-structural Morphological Filter}

Traditional morphological filter is typically to select only one structural element for background suppression. At the edge of clouds, image gray value is higher, and it likely contains noise which impacts the SNR of targets. To solve this problem, before the infrared image background suppression, the first step is to analyze the structural shapes of clouds edge, and then select a multiple structural element with the size and the direction associated with cloud edges. In morphological filter, we use a variety of different sizes and shapes of structural elements to do background suppression for infrared image under cloud background, using several structural elements for the image to conduct rot, expandable composition operation to achieve the best background suppression excellent results. Taking into account the infrared image influenced by light and other factors, image at the edge of the clouds and some interior region generally has high gray value. It is extremely easy to cause much noise which impacts the target' SNR during the process of small target background suppression. According to the specific structural features of the clouds and the correlation of the gray values of adjacent pixels, selecting the appropriate structural elements is very important to the infrared image morphological filter under cloud background. 


\section{Wavelet Threshold Method}

There are many methods of using wavelet for image background suppression. In this paper, we use the method of wavelet transform threshold background suppression to process clouds image, which is a method of image background suppression with a simple design and a better effect. In cloud background infrared image, most noises and clouds are in the low frequency domain, but the target is in the high frequency domain. To sum up, the wavelet coefficient of target is greater than the wavelet coefficient of clouds and noises. So, in the whole process of the wavelet transform, we need to select an appropriate threshold, in the processing of wavelet coefficient, to inhibit the small coefficients of contain noise and clouds interference, and to keep the large wavelet coefficients. Using the method of wavelet transform threshold background suppression, there are two very important factors closely related to the effect of image after suppression: one is the selection of threshold function; the other is the selection of threshold parameter. Threshold functions mainly include the hard threshold function and soft threshold function. The hard threshold function, although it has good inhibition effect, can better protect the image edge detail information, but the image reconstruction is prone to distortion. And for the soft threshold function, its process is relatively smoother, but the details in image edge are prone to distortion.

\section{Test results}

We use MATLAB to simulate several traditional background suppression methods, including the pulse median filter, the multi-structure morphological filter, the wavelet threshold method with the hard threshold function and the soft threshold function. The experimental results are seen in Fig. 1.

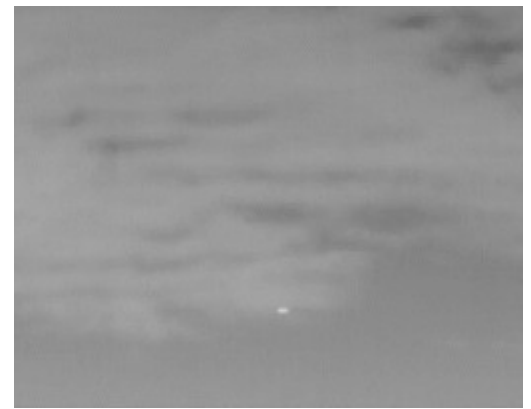

(a) Original image

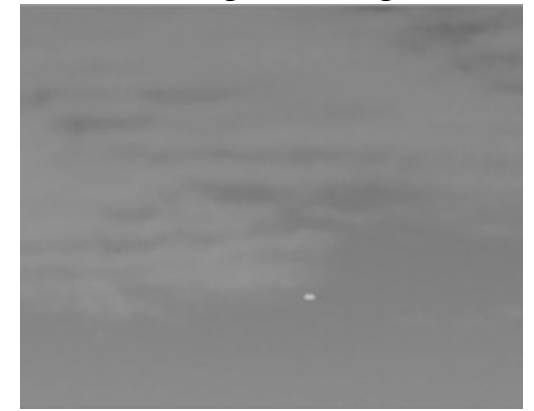

(c) Multi-structure morphological

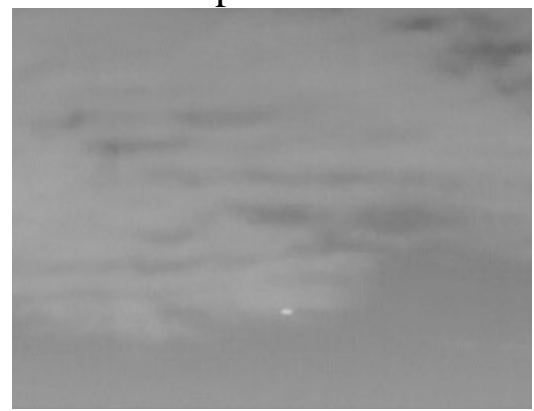

(b) Pulse median

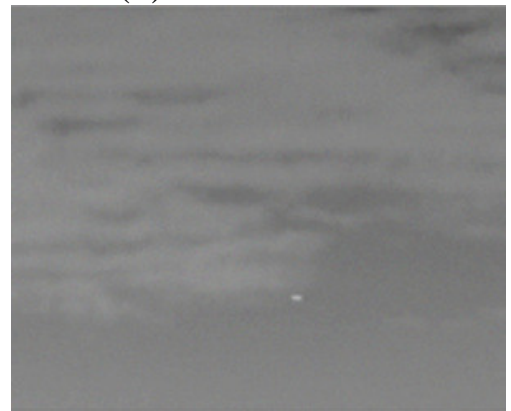

(d) Hard threshold function

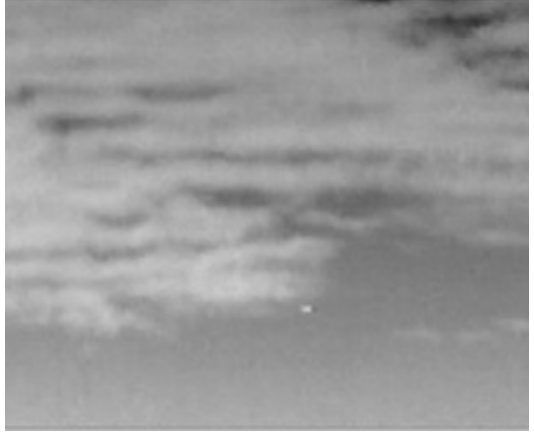

(e) Soft threshold function

Fig.1.The experimental results 
From the Fig. 1(b), we can find that pulse adaptive median filter algorithm for cloud background infrared image has a good inhibitory effect, and part pixels of the cloud background infrared image will be affected by impulse noise. Pulse median filter plays a significant inhibitory effect, but not good in image gray smooth and strong border areas.

In Fig.1(c), it shows that multi-structure morphological filter has a good background suppression effect, but part of the structural elements of small target easily be filtered out as noise.

The experimental results (Seen in Fig. 1(d) and Fig. 1(e).) show the effects of background suppression using wavelet threshold methods with the hard threshold function and the soft threshold function. The hard threshold function, as it has a good inhibitory effect, can well protect the image edge details and other information, but it is prone to distortion in the entire image. The soft threshold function is relatively smoother than the hard threshold function, but the information tends to be distorted at the edges of the image.

\section{Conclusion}

In this paper, the pulse median filter, the multi-structure morphological filter and wavelet threshold method have been simulated using MATLAB. As the experimental results shown, the pulse median filter has good inhibitory effect but not good in image gray smooth and strong border areas, the multi-structure morphological filter has some limitations for part of the structural elements of small target easily being filtered out as noise, and wavelet threshold method maybe result that the image information is incomplete and the SNR of the image is decreased.

\section{Acknowledgement}

In this paper, the research was sponsored by the Programs of the National Natural Science Foundation of China (Project No. 61263040 and No. 61162002), and the Scientific Research Foundation of Jiangxi Provincial Education Department in China (Project No. GJJ13528).

\section{References}

[1] Gonzalez R C, Woods R E. Digital Image Processing, 2nd Edition [M]. USA: Prentice Hall, 2003, 56-62.

[2] Handhold M M, Thomas D W. The Two Dimensional Adaptive (TDLMS) Algorithm [J]. IEEE Trans. Circ. Sys. 1988, 35(5):485-494.

[3] Jicheng Li, Zhenkang Shen. Small Moving Target Detection in Clutter Infrared Background [J]. Infrared and Laser Engineering, 1997, 26 (6): 8-13.

[4] Yaqiong Zeng, Qian Chen. Dim and Small Target Background SuppressionBased on Improved Bilateral Filtering for Single Infrared Image [J]. Infrared Technology, 2011, 33 (9): 537-540.

[5] Victor T T, Tamar P and so on. Morphology-based Algorithm for Point Target Detection in Infrared Backgrounds. Signal and Data Processing of Small Targets, Orlando, FL, USA, 1993.Proc. SPIE, 2005, 1954: 2-11.

[6] Rivest J F, Fortin R. Detection of Dim Targets in Digital Infrared Imagery by Morphological Image Processing [J]. Opt. Eng.. 1996, 35(7):1886-1893.

[7] Yang Wang, Qinbo Zheng, Junping Zhang. Real-time detection of small target in IR grey image based on mathematical morphology [J]. Infrared and Laser Engineering, 2003, 32 (1): 28-31.

[8] Zeng M, Li J X, Zhang P. The Design of Top-Hat Morphological Filter and Application to Infrared Target Detection [J]. Infrared Phys. \& Tech.. 2006, 48(1): 67-76.

[9] Bai X Z, Zhou F G, Jin T, Xie Y C. Infrared small Target Detection and Tracking under the Conditions of Dim Target Intensity and Clutter Background. MIPPR 2007:AutomaticTarget 
Recognition Imand age Analysis and Multispectral Image Acquisition, Wuhan, China,2007 ,Proc. of SPIE, 6786: 67862M.

[10] Bai X Z, Zhou F G, Xie Y C. New Class of Top-Hat Transformation to Enhance Infrared Small Targets [J]. Journal of Electronic Imaging, 2008, 17(3):030501-1-3.

[11] Vincent L. Morphological Grayscale Reconstruction in Image Analysis: Application and Efficient Algorithms [J]. IEEE Trans. On Image Processing, 1993, 2(2):176-201.

[12] Pesaresi M, Benedikt J. A New Approach for the Morphological Segmentation of Highresolution Satellite Imagery [J]. IEEE Transactions on Geoscience and remote sensing, 2001, 39(2):309-320.

[13] Braga-Neto U, Goutsias J. Automatic Target Detection and Tracking in Forward-looking Infrared Image Sequences using Morphological Connected Operators [J]. Electronic Imaging, 2004, 13(4):802-813.

[14] Mallat S and Zhang S. Characterization of Signals from multiscale edges [J]. IEEE Trans on PAMI, 1992, 7,147(7):710-732.

[15] Donoho D L and Sotue I M J. Ldeals Patialada Ptation via wavelet shrink age [J]. Biometrika. Ctrika 1994,81,425-102.

[16] Donoho D L and Sotue I M J. Adapting tounk llowns moothness via wavelet shrinkage [J]. Joumal of American Stat. Assoe,1995, 90(432):1200-1224.

[17] Candes E J, Donoho D L. Dgelets:A key to higher dimension alintemittency [J]. Philans R SoeLond A,1999,357:2495-2509.

[18] Chang S G, Yu B, Vetter M. Adaptive wavelet thresholding for image denoising and compression [J]. IEEE Trans. On IP, 2000,9(9):1532-1546.

[19] Luisier F, Blu T, Unser M. A new SURE approach to image denoising: interscal orthonormal wavelet thresholding [J]. IEEE Trans.Image Processing, 2007, 16(3):593-60. 